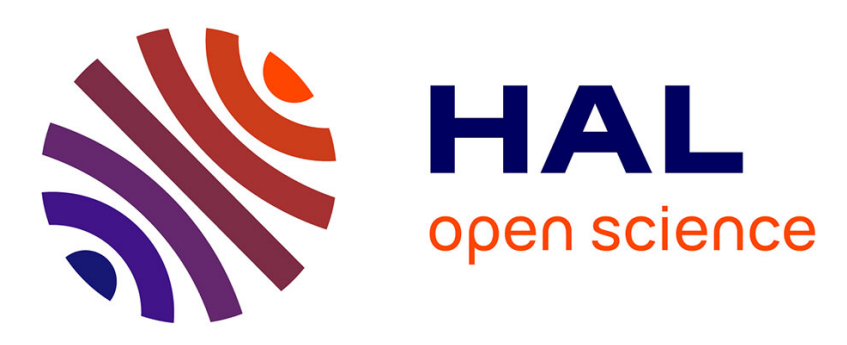

\title{
Thresholds of Envelope Soliton Formation for Dipole-Exchange Spin Waves in Yttrium-Iron Garnet Films
}

\author{
A. Slavin, H. Benner, K. Foos, T. Lesperance
}

\section{- To cite this version:}

A. Slavin, H. Benner, K. Foos, T. Lesperance. Thresholds of Envelope Soliton Formation for DipoleExchange Spin Waves in Yttrium-Iron Garnet Films. Journal de Physique IV Proceedings, 1997, 07 (C1), pp.C1-459-C1-460. 10.1051/jp4:19971187 • jpa-00254836

\section{HAL Id: jpa-00254836 https://hal.science/jpa-00254836}

Submitted on 1 Jan 1997

HAL is a multi-disciplinary open access archive for the deposit and dissemination of scientific research documents, whether they are published or not. The documents may come from teaching and research institutions in France or abroad, or from public or private research centers.
L'archive ouverte pluridisciplinaire HAL, est destinée au dépôt et à la diffusion de documents scientifiques de niveau recherche, publiés ou non, émanant des établissements d'enseignement et de recherche français ou étrangers, des laboratoires publics ou privés. 


\title{
Thresholds of Envelope Soliton Formation for Dipole-Exchange Spin Waves in Yttrium-Iron Garnet Films
}

\author{
A.N. Slavin, H. Benner*, K.J. Foos* and T. Lesperance \\ Physics Department, Oakland University, Rochester, MI-48309, U.S.A. \\ * Technische Hochschule Darmstadt, 64289 Darmstadt, Germany
}

\begin{abstract}
The power thresholds of spin wave envelope soliton formation from input rectangular radio-pulses of duration $T$ in a thin yttrium-iron garnet (YIG) film were studied experimentally. The measurements were done for strongly dispersive dipoleexchange spin waves in the vicinity of one of the dipole gaps in the spectrum of a film with strongly pinned surface spins. The observed threshold curve $P_{\mathrm{th}}=\mathrm{f}\left(1 / \mathrm{T}^{2}\right)$ is non-monotonous and has a fine structure consisting of three minima corresponding to the formation of $n=1,2$, and 3 solitons. The observed shape of the threshold curve is explained by the theory of envelope soliton formation where dissipation in the medium is taken into account. The values and positions of the minima on the experimentally measured threshold curve are determined by the dissipation parameter $\Gamma=\gamma \Delta H$ which is proportional to the half-linewidth $\Delta H$ of ferromagnetic resonance in the experimental YIG film.
\end{abstract}

\section{INTRODUCTION}

Envelope solitons are stable nonlinear wave pulses that can propagate in nonlinear dispersive media without changing their shape. The classical theory of envelope soliton formation from rectangular input pulses of duration $T$ in a lossless medium predicts that the threshold of soliton formation is proportional to $I / T^{2}$ [1]. The experimental data obtained for spin wave envelope solitons in magnetic films [2,3] is , for the most part, in good qualitative agreement with this prediction. However, recent experimental measurements of soliton formation thresholds for low-dispersive backward volume magnetostatic waves (BVMSW)[4] have shown qualitative disagreements between the classical theory [1] and the experiment. The threshold curve $P_{t h}=f\left(I / T^{2}\right)$ experimentally observed in [4] was non-monotonous and had a fine structure consisting of two minima that correspond to the formation of $n=1$ and $n=2$ envelope solitons. The above described fine structure of the soliton threshold curve was explained in [5], where dissipation in the medium was taken into account. The resulting expression for a normalized soliton threshold in a weakly dissipative medium has the form

$$
P_{t h} / P_{t h_{n}}^{\min }=\left[1+\left(T_{n} / T\right)^{4}\right] /\left[2\left(T_{n} / T\right)^{2}\right]
$$

The threshold curve Eq.(1) has $n$ minima at $T=T_{n}$. The value of optimum input pulse duration $T_{n}$ corresponding to the minimum threshold of formation of $1,2, \ldots n$ solitons are given by

$$
T_{n}=\left[\pi^{2}(2 n-1)^{2}|D| /\left(2 \Gamma v_{g}^{2}\right)\right]^{\frac{1}{2}}
$$

where $v_{\mathrm{g}}$ and $D$ are group velocity and dispersion calculated at a carrier wave number $k_{0}$ of the input pulse, while $\Gamma$ is the dissipation parameter. Equations (1) and (2) give good quantitative explanation to the threshold curves of envelope soliton formation for low-dispersive BVMSW experimentally observed in [4].

The aim of our present paper is to study experimentally the thresholds of envelope soliton formation for highly dispersive dipole-exchange spin waves in thin YIG films, and to check the theory of envelope soliton formation in weakly dissipative medium, Eq.(1), in the dipole-exchange spin wave spectrum, where the values of group velocity $v_{\mathrm{g}}$ and dispersion $D$ differ substantially from the corresponding values in a purely dipolar (magnetostatic wave) spectrum [3,4].

\section{EXPERIMENT}

For our experiments we used a sample of a perpendicularly magnetized $\left(H_{e}=3140 \mathrm{Oe}\right)$ monocrystal YIG film of the crystallographic orientation [111], thickness $L=4.6 \mu \mathrm{m}$, static magnetization $4 \pi M_{0}=1750 \mathrm{G}$, and ferromagnetic resonance linewidth $2 \Delta H=0.7$ Oe (dissipation parameter $\Gamma=\gamma \Delta H=6 \cdot 10^{6} \mathrm{~s}^{-1}$ ). The sample was placed in the prototype delay line consisting of two microstrip transducers (width $=410^{-3} \mathrm{~cm}$, length $=0.35 \mathrm{~cm}$, distance between transducers $l=0.7 \mathrm{~cm}$ ) [6]. Measurements were made at a room temperature. The carrier frequency $\left(\omega_{0} / 2 \pi=3.764 \mathrm{GHz}\right)$ of input rectangular pulses of variable duration $(0.3 \mu \mathrm{s}<T<2.7 \mu \mathrm{s})$ has been chosen on the left slope of the fifth dipole gap in the film spectrum (see Fig. 1) where the group velocity was $V_{g}=5.710^{5} \mathrm{~cm} / \mathrm{s}$ and the dispersion was $D=-10^{5} \mathrm{~cm}^{2} / \mathrm{s}$. 


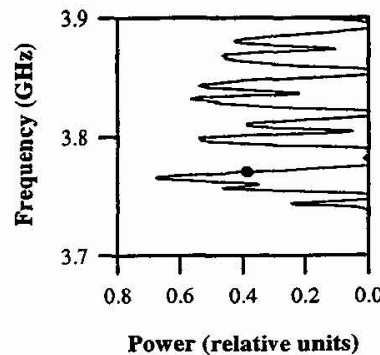

a)

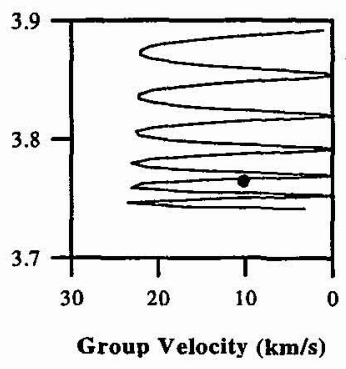

b)

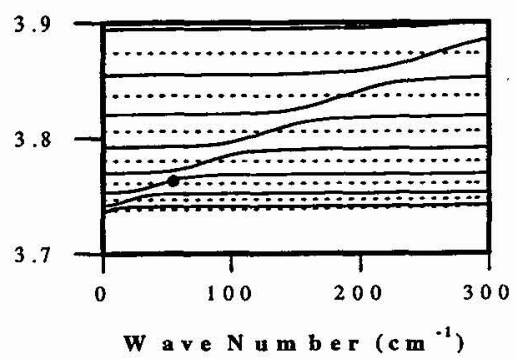

c)

Figure 1: (a) Experimental frequency dependence of the output power of the experimental delay line, measured in the linear regime under continuous excitation, (b) calculated group velocity, and (c) dipole-exchange frequency spectrum of spin waves in the investigated YIG film. The operating point on the left slope of the dipole "gap" in the spectrum, where measurements of threshold of soliton formation were done, is shown by a dot.

We measured dependences of the peak power of the output pulse on the input pulse power for different input pulse durations. Threshold power of soliton formation was determined (similarly to $[2,4]$ ) as a level of the input power at which the peak output power starts to deviate from the linear trend. The shape of the obtained threshold curve $P_{\text {th }}=f\left(1 / T^{2}\right)$ was similar to that of the curve obtained in [4] for BVMSW. However, in the dipole-exchange case the threshold curve had three minima corresponding to the formation of envelope solitons of the orders $n=1,2,3$. The results of comparison of experimentally measured and theoretically calculated (using Eq.(1)) soliton thresholds for highly dispersive dipole-exchange spin waves are presented in Fig.2. We used the minimum value of the threshold for a single soliton formation ( $P_{t h_{1}}^{\min }$ at $T=T_{1}=0.5 \mu \mathrm{s}$ ) to normalize the experimental threshoid curve. It is clear from Fig.2 that the theory Eq.(1), which takes into account dissipation in the YIG film, is in good qualitative agreement with the experiment. In the region of relatively short $(0.3 \mu \mathrm{s}<T<0.7 \mu \mathrm{s})$ input pulses, where a single $(n=1)$ envelope soliton is formed we can even claim a good quantitative agreement between the theory and experiment.

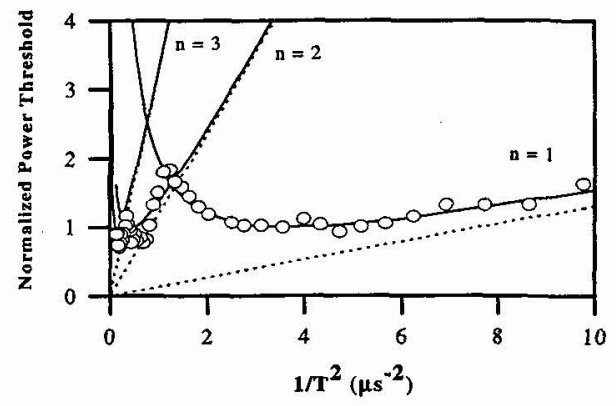

a)

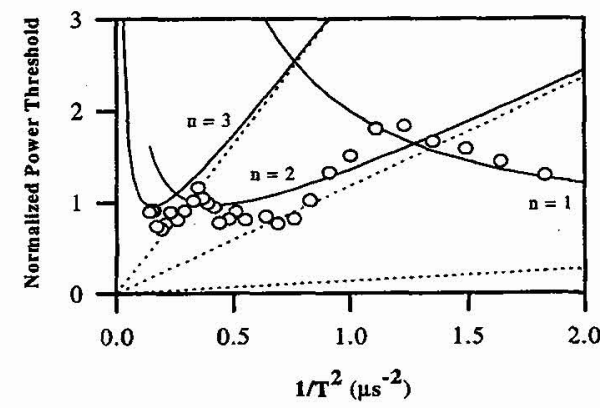

b)

Figure 2: (a) Comparison of normalized soliton thresholds theoretically calculated using Eq.(1) (solid lines) and experimentally measured for solitons of dipole-exchange spin waves in the operating point shown in Fig.1; (b) is a magnification of (a) for the region of relatively long input pulses $0.7 \mu \mathrm{s}<T<2.7 \mu \mathrm{s}$.

\section{Acknowledgments}

This work was supported in part by the Grant CC-3401 from the Research Corporation, and as a project of SFB 185 "Nichtlineare Dynamik" by the Deutsche Forschungsgemeinschaft.

\section{References}

[1] Zvezdin A.K. and. Popkov A.F, Sov.Phys.JETP 57 (1983) 350-357.

[2] De Gasperis P., Marcelli R., and Miccoli G., Phys Rev. Lett. 59(1987) 481-484.

[3] Chen M., Tsankov M.A., Nash J.M., and Patton C.E., Phys.Rev.B 49 (1994) 12773-12790.

[4] Nash J.M., Patton C.E., and Kabos P., Phys.Rev.B 51 (1995) 15079-15084.

[5] Slavin A.N., Abstracts of the MMM-95 Conference, Abstract DD-02, pp. 212-213, Philadelphia, 1996.

[6] Kalinikos B.A., Kovshikov N.G., and Slavin A.N., Sov.Phys. JETP 67 (1988) 303-312. 